\title{
DEMOGRAFÍA Y DESARROLLO OVÁRICO DE ANASTREPHA DISTINCTA (DIPTERA: TEPHRITIDAE) EN SU HOSPEDERO NATURAL INGA SPURIA (FABACEAE) Y EN MANGO (MANGIFERA INDICA L.) INFESTADO EN CONDICIONES DE LABORATORIO
}

\section{Azucena OROPEZA-CABRERA, ${ }^{1}$ Pablo LIEDo, ${ }^{1}$ Emilio HERNÁNDEZ ${ }^{2}$ y Jorge TOLEDO ${ }^{1, *}$}

\author{
${ }^{1}$ El Colegio de la Frontera Sur. Tapachula, Chiapas, México. C.P. 30700. México. \\ ${ }^{2}$ Programa Moscafrut, SAGARPA-IICA. Subdirección de Desarrollo de Métodos. Camino a los Cacaotales S/N. \\ Metapa de Domínguez, Chiapas, C. P. 30860. México. <jtoledo@ecosur.mx>
}

Recibido: 03/05/2013; aceptado: 21/04/2015

Oropeza-Cabrera, A., Liedo. P., Hernández, E. \& Toledo, J. 2015. Demografía y desarrollo ovárico de Anastrepha distincta (Diptera: Tephritidae) en su hospedero natural Inga spuria (Fabaceae) y en mango (Mangifera indica L.) infestado en condiciones de laboratorio. Acta Zoológica Mexicana (n. s.), 31(2): 149158.

RESUMEN. Anastrepha distincta (Greene) es una especie de tefrítido que en condiciones naturales se le encuentra infestando frutos de plantas del género Inga. Aunque en raras ocasiones se le ha reportado infestando especies de frutos comerciales, existen dudas si esos registros se deben a errores de identificación o si ésta especie realmente tiene el potencial para desarrollarse en esos frutos. En este trabajo se evaluó la capacidad de ésta especie para desarrollarse en frutos de mango (Mangifera indica L.) bajo condiciones de laboratorio. Las moscas utilizadas fueron recuperadas como larvas infestando frutos de "cuajinicuil" (Inga spuria Humb. \& Bonpl. ex Willd.) en condiciones naturales. Se expusieron frutos de mango de las variedades "coche” y "Ataulfo" a grupos de adultos promoviendo su infestación bajo condiciones de laboratorio. Con un enfoque demográfico se determinó la sobrevivencia, capacidad reproductiva y desarrollo ovárico de la progenie obtenida. Anastrepha distincta fue capaz de infestar frutos de mango y tener un desarrollo exitoso bajo estas condiciones. La expectativa de vida de los machos fue de 110.9, 107.9 y 114.3 días, en tanto que para las hembras fue de 107.6, 69.0 y 132.3 días cuando provenieron de mango "Ataulfo", mango "coche”, e I. spuria, respectivamente. La fecundidad bruta $\left(\Sigma \mathrm{m}_{\mathrm{x}}\right)$ de las hembras fue de 392.5, 401.1 y 176.1 huevos/hembra, y la fecundidad neta $\left(\Sigma \mathrm{l}_{\mathrm{x}} \mathrm{m}_{\mathrm{x}}\right)$ fue de 349.2, 244.4 y 168.6 huevos/hembra, en mango “Ataulfo”, mango “coche” e I. spuria, respectivamente. Las hembras obtenidas de I. spuria registraron mayor número de ovariolas, mientras que los mayores porcentajes de eclosión de huevos se observaron en las hembras recuperadas de mango "coche”. Se discuten las posibles causas de que el mango no sea un hospedero natural de $A$. distincta a pesar de que esta especie puede desarrollarse y reproducirse en esos frutos bajo condiciones de laboratorio.

Palabras clave: Fecundidad, fertilidad, longevidad, moscas de la fruta, hospederos.

\section{INTRODUCCIÓN}

La "mosca de las ingas”, Anastrepha distincta Greene es una especie con amplia distribución en el continente americano. Es de poca importancia económica debido a su asociación con frutos del género Inga (Fabaceae), los cuales no son aprovechados comercialmente (Hernández-Or-
Oropeza-Cabrera, A., Liedo. P., Hernández, E. \& Toledo, J. 2015. Demography and ovarian development of Anastrepha distincta (Diptera: Tephritidae) in its natural host Inga spuria (Fabaceae), and mango (Mangifera indica L.) infested under laboratory conditions. Acta Zoológica Mexicana (n. s.), 31(2): 149158.

ABSTRACT. Anastrepha distincta (Greene) is a tephritid species that under natural conditions is found infesting fruits of plants of the genus Inga. Although rarely it has been reported infesting comercial fruits, it is doubtful whether these records were misidentifications or if this species really has the potential to develop in these fruits. In this study we evaluated whether this species is able to develop on mango fruits (Mangifera indica L.). The flies used for experiments were obtained as larvae infesting fruits of “cuajinicuil” (Inga spuria Humb. \& Bonpl. ex Willd.) under natural conditions. Fruits of mango of the "coche” and "Ataulfo" cultivars were exposed to groups of adult flies to promote their infestation under laboratory conditions. With a demographic approach, the offspring survival, reproductive capacity and ovarian development were determined. Anastrepha distincta was capable to infest mango fruits and successfully develop under lab conditions. Male life expectancy was 110.9, 107.9 and 114.3 days for "Ataulfo" mango, "coche" mango and I. spuria, respectively; in females it was 107.6, 69.0 and 132.3 for the same hosts. Gross fecundity $\left(\Sigma m_{x}\right)$ was 392.5, 401.1 and 176.1 eggs/female, and net fecundity $\left(\Sigma \mathrm{l}_{\mathrm{x}} \mathrm{m}_{\mathrm{x}}\right)$ was 349.2, 244.4 and 168.6 eggs/female, for the females recovered from mango cultivars "Ataulfo", and "coche”, and I. spuria, respectively. The females recovered from $I$. spuria showed the greatest number of ovarioles, while the greatest percentages of egg hatching were obtained from females reared on mango "coche". The possible causes that mango is not a natural host for $A$. distincta, even though this fly species can successfully develop and reproduce in mango fruits under laboratory conditions are discussed.

Key words: Fecundity, fertility, longevity, fruit flies, hosts.

tiz 1992). Esta especie se relaciona filogenéticamente en el grupo fraterculus, que incluye varias especies plaga de diversos frutales con importancia económica (Norrbom et al. 1999). Se ha reportado que esta especie tiene potencial para infestar frutos de importancia económica como la naranja (Citrus sinensis L.) y el mango (Mangifera indica L.) (Aluja 1994), pero se ha discutido que estos registros 
pudieran tratarse de indentificaciones erróneas (Norrbom \& Kim 1988). Surgen entonces las siguientes preguntas: 1) ¿Tiene A. distincta el potencial de desarrollarse en frutos de plantas diferentes al género Inga? De ser así, 2) ¿Podría esta especie ampliar su rango de hospederos si se reduce la disponibilidad de sus hospederos naturales?; o ¿Podrían incrementarse sus poblaciones por cambios climáticos favorables, tal y como está ocurriendo con otras especies de tefrítidos? (Ni et al. 2012).

El enfoque demográfico puede ser utilizado para determinar el efecto de diferentes hospederos en las tasas de crecimiento poblacional. Se ha encontrado que la elección de especies hospederas afecta el tiempo de desarrollo, la sobrevivencia y la fecundidad, y como consecuencia de ello el crecimiento de las poblaciones (Carey 1984, Krainacker et al. 1987, Celedonio-Hurtado et al. 1988, Liedo \& Carey 1996).

El objetivo de este trabajo fue realizar un análisis demográfico de A. distincta, comparando el potencial reproductivo de las hembras procedentes de un hospedero silvestre infestado naturalmente, con hembras procedentes de dos variedades de mango infestados en condiciones de laboratorio.

\section{MATERIALES Y MÉTODOS}

Material biológico. El material biológico de A. distincta fue obtenido a partir de larvas en frutos infestados de “cuajinicuil” (Inga spuria Humb. \& Bonpl. ex Willd.) colectados en la zona alta ( 820 msnm) de la Región del Soconusco, Chiapas ( $\left(5^{\circ} 02^{\prime} 11^{\prime \prime} \mathrm{N}\right.$; 92 $\left.05^{\circ} 64^{\prime \prime} \mathrm{O}\right)$ ). Los frutos recolectados fueron trasladados al laboratorio de El Colegio de la Frontera Sur (ECOSUR) (Tapachula, Chiapas). Ahí fueron contados y pesados en una balanza de precisión (Ohaus, Pine Brook, NJ) y colocados en charolas de plástico en donde permanecieron durante seis días (26 $\pm 1{ }^{\circ} \mathrm{C}, 75 \pm 5 \%$ de H.R.; fotoperiodo de 12:12 h luz: oscuridad), hasta que las larvas alcanzaron el tercer estadio. Posteriormente, los frutos fueron abiertos para extraer las larvas, colocándolas en contenedores de plástico (15 cm por lado $\times 6 \mathrm{~cm}$ de alto) con vermiculita húmeda (Strong-lite fine, Sungro Horticulture, Seneca, IL, USA), para promover la pupación. Las pupas permanecieron en los contenedores por 13 días, dos días antes de la emergencia fueron separadas de la vermiculita con un tamiz (malla 18) y se colocaron en jaulas de vidrio de $27 \mathrm{dm}^{3}$ para la emergencia de los adultos. Posteriormente fueron separados por sexo y transferidos a jaulas de las mismas dimensiones con alimento permanente que consisitió en una mezcla seca de levadura hidrolizada enzimáticamente (MP Biomedicals, Irvine, CA) más sacarosa en una proporción de 1:3, colocada en tapas de cajas Petri de $10 \mathrm{~cm}$ de diámetro, y agua suministrada en viales de $50 \mathrm{ml} \mathrm{cu-}$ biertos con algodón. La determinación específica se realizó mediante el uso de las claves taxonómicas de especies de Anastrepha en México (Hernández-Ortiz 1992).

Para los experimentos de infestación de frutos de mango (Mangifera indica L.), se recolectaron frutos de dos variedades, "coche" y "Ataulfo" en un huerto aledaño a Tapachula, Chiapas, México (1454’27” N; 92¹6’49” O). Estas variedades fueron seleccionadas debido a que bajo condiciones de campo, el mango "coche" es susceptible a la infestación por A. ludens y A. obliqua, mientras que el mango cv. "Ataulfo" es más infestado por $A$. ludens y rara vez es atacado por A. obliqua, con excepción del llamado “mango niño" (Aluja et al. 2014). Los frutos fueron cosechados en estado de madurez comercial y lavados con agua de la llave durante 30 minutos antes de realizar la infestación. Todos los frutos fueron inspeccionados al exterior para verificar que estuvieran libres de piquetes de oviposición. Cuando las moscas alcanzaron la madurez sexual, se introdujeron cinco frutos por jaula (30 $\times 30 \times 30 \mathrm{~cm}$ ) junto con 50 parejas de moscas (relación 10 parejas por fruto) para cada variedad. Los frutos estuvieron expuestos a la infestación durante $12 \mathrm{~h}$ continuas para obtener un nivel promedio de infestación de 25 larvas por fruto. Posteriormente fueron retirados de las jaulas y colocados en charolas de plástico previamente etiquetadas, en un cuarto con condiciones controladas (temperatura $26^{\circ} \mathrm{C}$ y H.R. 70\%), en donde permanecieron por un período de 14 días para permitir el desarrollo larvario. Una vez que las larvas alcanzaron el tercer estadio (14 días después de la infestación), los frutos fueron abiertos para extraer y cuantificar el número de larvas desarrolladas. Las larvas recuperadas se colocaron en un contenedor de plástico con vermiculita húmeda ( $60 \%$ de HR) para promover la pupación, en donde permanecieron por 13 días aproximadamente.

Longevidad y supervivencia. Los adultos obtenidos de los diferentes frutos fueron utilizados para realizar un estudio demográfico. Se utilizaron jaulas de vidrio $(10 \times 10$ $\times 10 \mathrm{~cm}=1 \mathrm{dm}^{3}$ ), colocando una pareja (hembra y macho) por jaula provistas con alimento y agua, en un total de 30 jaulas por cada tratamiento o fruto hospedero.

Fecundidad. El potencial reproductivo se determinó mediante la estimación de la fecundidad de las hembras, después que los adultos se aparearon. A partir del octavo día se colocó un dispositivo de oviposición en cada una de las jaulas con las dimensiones descritas previamente. Estos dispositivos consistieron en esferas de agar (Boller 1968) preparadas disolviendo $80 \mathrm{~g}$ de agar en tres litros de agua y calentando la mezcla hasta ebullición, a la cual se le añadieron $5 \mathrm{ml}$ de colorante verde vegetal (McCormick de México, S.A. de C.V.), la mezcla se vació en moldes 
esféricos de 2 cm de diámetro y una vez que se gelatinizó, fueron envueltas con papel Parafilm (American Nacional Can TM, Nena, Wi). Se colocó una esfera en cada jaula de $1 \mathrm{dm}^{3}$. Las esferas fueron remplazadas cada $24 \mathrm{~h}$, las que se retiraban de las jaulas fueron disectadas para extraer y cuantificar el número de huevos ovipositados por día, actividad que se realizó hasta que murió la última hembra. También se llevó un registro diario del número de oviposturas y huevos por ovipostura por hembra.

Fertilidad. La fertilidad de los huevos se determinó alineando diariamente una muestra de 50 huevos sobre un recorte de tela negra colocada sobre una esponja húmeda dentro de una caja de Petri. Las muestras fueron incubadas en un cuarto a $26{ }^{\circ} \mathrm{C}$ y $70 \%$ HR, y después de seis días se cuantificó él número de huevos no eclosionados y por diferencia se estimaron las larvas eclosionadas, que expresado en porcentaje correspondió a la fertilidad.

Desarrollo ovárico. Para estimar el desarrollo ovárico de las hembras criadas en cada fruto, se seleccionaron pupas que registraron un peso de 11.0 a $14.0 \mathrm{mg}$, con el fin de evitar el posible sesgo por el tamaño, y se colocaron en tapas de cajas de Petri dentro de una jaula de vidrio cúbica de $27 \mathrm{dm}^{3}$ para que emergieran los adultos. Después que emergieron, cada cuatro días se tomaron al azar seis hembras de cada tratamiento, se separó el abdomen del resto del cuerpo de las hembras y se extrajo el ovario izquierdo. Cada ovario se acomodó sobre un portaobjeto para medir su longitud y el ancho. Las mediciones se realizaron adaptando una reglilla micrométrica de un micrómetro al ocular de un microscopio estereoscopio marca Carl Zeiss SV 26. También se cuantificó el número de ovariolas por ovario, actividad que se realizó desde el día uno hasta que las moscas alcanzaron 31 días de edad.

Análisis de datos. Los valores de supervivencia y reproducción de las hembras criadas en los tres diferentes frutos fueron sujetos a un análisis demográfico de acuerdo al método propuesto por Carey (1993). Para el cálculo de los parámetros de población se consideró un tiempo de desarrollo de huevo a adulto de 31 días y una sobrevivencia de $30 \%$, con base en las observaciones que se realizaron durante el desarrollo de los inmaduros y lo reportado para otras especies del mismo género (Celedonio-Hurtado et al. 1988). Para determinar si había diferencias entre los valores de fecundidad, fertilidad, largo y ancho de ovarios, así como del total de ovariolas se hizo un análisis de varianza (ANOVA) utilizando datos transformados a la raíz cuadrada de $x+0.5$ para cumplir con la normalidad de acuerdo con la prueba de Shapiro-Wilk (W = 0.7142, $P<0.001)$ y homogeneidad de varianzas con base en la prueba de Bartlett $\left(\chi_{\mathrm{K}-1}^{2}=22.236\right.$, g.l. $\left.=4, \mathrm{P}=0.0002\right)$. La separación de medias se hizo con la prueba de Tukey (SAS Institute 1999, R Core Team 2014).

\section{RESULTADOS}

Longevidad y supervivencia. Las hembras recuperadas de los tres frutos presentaron diferencias significativas ( $F$ $=59.59$; g.l. $=2,573 ; P<0.001)$ en su supervivencia (Fig. 1 A). La mayor supervivencia se observó en hembras recuperadas de su hospedero natural I. spuria, seguida de las hembras procedentes de mango "Ataulfo". Sin embargo, a partir de los 175 días de edad, el 20\% de las hembras que registraron la mayor longevidad fueron las provenientes de mango "Ataulfo". Las hembras procedentes de mango "coche" presentaron una menor supervivencia desde la emergencia, alcanzando el 100\% de mortalidad a los 150 días de edad. La supervivencia de los machos recuperados de los tres hospederos fue muy similar hasta los 121 días de edad, a partir de ésta edad la supervivencia de los machos procedentes de $I$. spuria fue mayor con diferencias significativas $(F=59.61$; g.l. $=2$, 615; $P<0.001$ ) (Fig. 1 B). Tanto las hembras como los machos de $I$. spuria presentaron una mayor expectativa de vida a la emergencia (edad cero), en comparación con la observada en adultos de ambos sexos recuperados de mango "coche” y "Ataulfo" (Cuadro 1).

Fecundidad. Los resultados indicaron que en condiciones controladas y en ausencia de su hospedero natural, $A$. distincta fue capaz de infestar frutos de mango "coche" y "Ataulfo" y completar exitosamente su ciclo biológico. El peso promedio de las pupas provenientes de frutos de I. spuria fue de $11.2 \pm 0.14 \mathrm{mg}$, en mango "coche" fue de $11.3 \pm 0.10 \mathrm{mg}$, y en pupas procedentes de mango "Ataulfo" promediaron $12.6 \pm 0.21 \mathrm{mg}$.

La mayor fecundidad bruta $\left(\Sigma \mathrm{m}_{\mathrm{x}}\right)$ se registró en hembras criadas en mango "coche”, en segundo lugar estuvieron las hembras recuperadas de mango "Ataulfo" y por último fueron las hembras criadas en su hospedero natural I. spuria. En cuanto a la fecundidad neta $\left(\Sigma \mathrm{l}_{\mathrm{x}} \mathrm{m}_{\mathrm{x}}\right)$, las hembras provenientes de mango "Ataulfo" presentaron la tasa más alta, seguidas de las hembras de mango "coche" y la mas baja se presentó en las hembras provenientes de I. spuria (Cuadro 1). Las diferencias observadas entre los valores de la distribución diaria de la fecundidad bruta y neta fueron significativas ( $\mathrm{F}=14.8$; g.l. $=2$, 258; $\mathrm{P}<$ 0.001) (Figs. 2A y 2B).

El número de huevos por ovipostura observado con mayor frecuencia fue de 1 a 3 huevos. Las hembras obtenidas de mango "Ataulfo" pusieron con mayor frecuencia un solo huevo por ovipostura. Las hembras provenientes de los otros dos hospederos registraron frecuencias similares para 1 y 2 huevos por ovipostura. Los casos de 3 a 10 huevos por ovipostura fueron raros (Fig. 3).

Fertilidad. La mayor eclosión de huevos fue observada en hembras procedentes de mango "coche”, mientras 

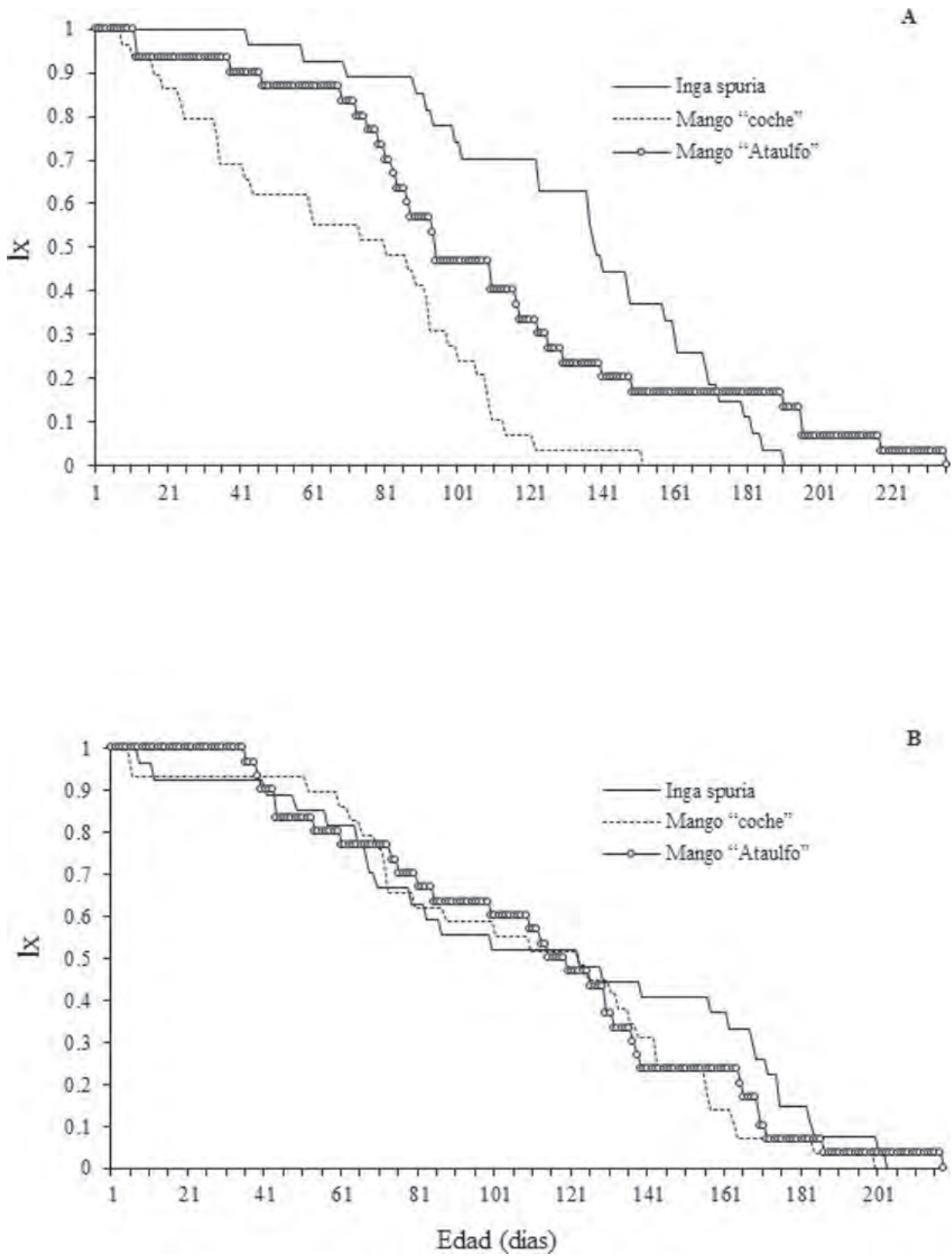

Figura 1. Supervivencia de hembras (A) y machos (B) de Anastrepha distincta criados en su hospedero natural Inga spuria y en frutos de mango bajo condiciones de laboratorio $(N=29$ parejas/tratamiento).

Cuadro 1. Fecundidad bruta, fecundidad neta (huevos por hembra) y expectativa de vida a la emergencia (edad cero) de Anastrepha distincta criadas en su hospedero natural Inga spuria y en frutos de mango bajo condiciones de laboratorio.

\begin{tabular}{lccccr}
\hline \multicolumn{1}{c}{ Hospederos } & $N$ & $\sum \mathbf{m}_{\mathbf{x}}$ & $\sum \mathbf{I}_{\mathbf{x}} \mathbf{m}_{\mathbf{x}}$ & \multicolumn{2}{c}{$e_{\theta}$} \\
\cline { 5 - 6 } & & & & \multicolumn{1}{c}{$\uparrow$} \\
\hline Mango “coche” & 29 & 401.1 & 244.4 & 107.9 & 69.0 \\
Mango “Ataulfo" & 30 & 392.5 & 349.2 & 110.9 & 107.6 \\
Inga spuria & 27 & 176.1 & 168.6 & 114.3 & 132.3 \\
\hline
\end{tabular}

que la menor eclosión se presentó en hembras de mango "Ataulfo" (Cuadro 2). Las diferencias observadas entre los valores de fertilidad fueron significativas $(F=59.09$; g.l. $=2$, 166; $P<0.001)$. La máxima fertilidad se observó cuando las hembras tenían entre 17 y 40 días de edad.

Parámetros de población. Los parámetros de población obtenidos para cada tratamiento indicaron que la tasa intrínseca de crecimiento fue mayor en las dos variedades de mango en comparación con el hospedero natural, I. spuria. El tiempo de generación fue menor para las hembras 

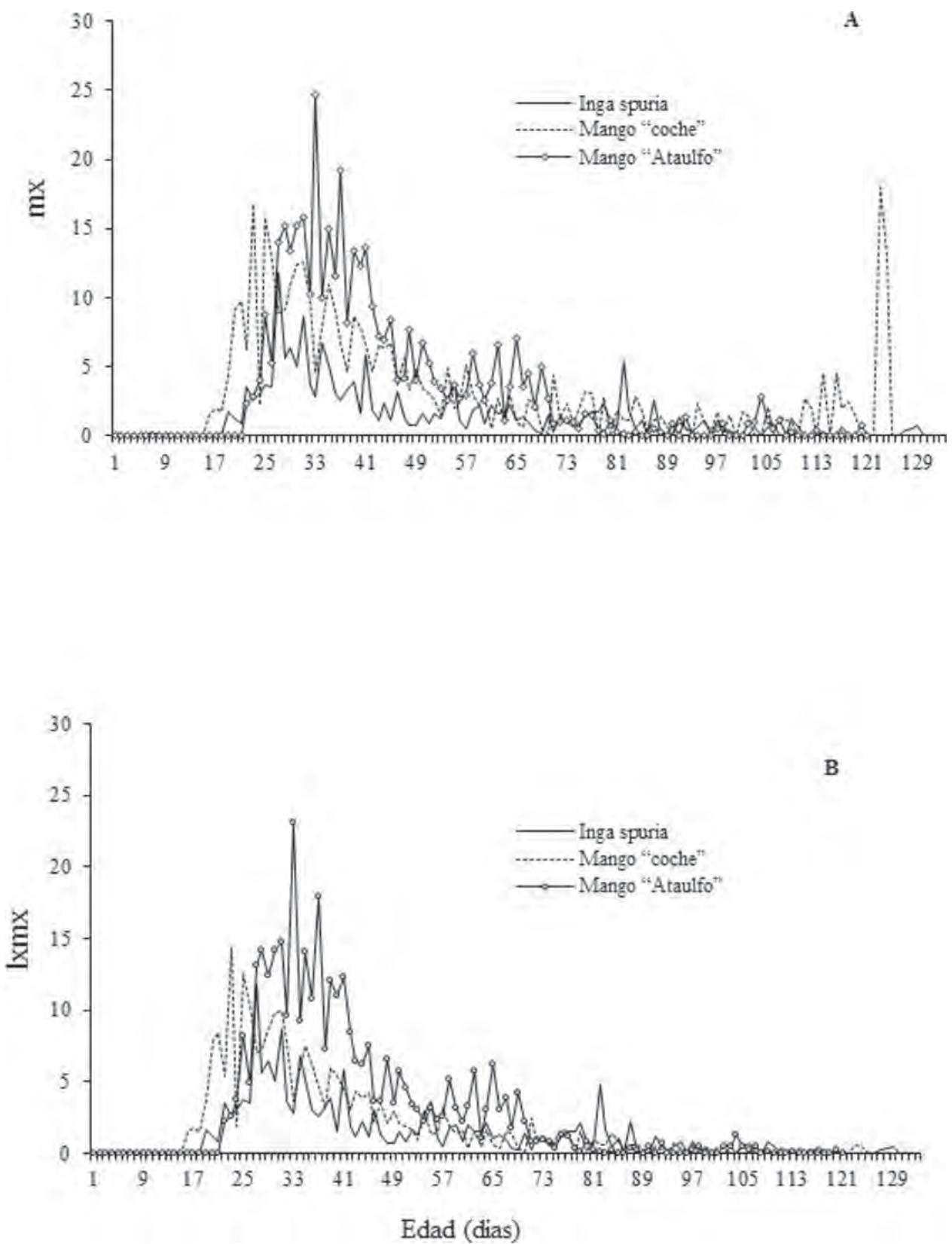

Figura 2. Fecundidad bruta (A) y neta (B) de Anastrepha distincta criadas en su hospedero natural Inga spuria y en frutos de mango bajo condiciones de laboratorio $(N=29$ parejas/tratamiento $)$.

Cuadro 2. Fertilidad de huevos (\% \pm e. e.) obtenidos de hembras de Anastrepha distincta criadas en su hospedero natural Inga spuria y en frutos de mango bajo condiciones de laboratorio.

\begin{tabular}{lrc}
\hline \multicolumn{1}{c}{ Hospederos } & $\boldsymbol{N}$ & Eclosión $(\% \pm$ e.e.) \\
\hline Mango “coche” & 5,924 & $33.22 \pm 4.3 \mathrm{a}$ \\
Mango “Ataulfo” & 10,085 & $24.71 \pm 1.4 \mathrm{~b}$ \\
Inga spuria & 3,954 & $29.54 \pm 3.2 \mathrm{ab}$ \\
\hline
\end{tabular}

provenientes de mango "coche”, seguido de las de mango "Ataulfo" y el mayor tiempo fue para en las de I. spuria. Lo anterior mostró que el tiempo en que se duplica la población fue similar entre las dos variedades de mango, pero en el hospedero natural fue 20\% mayor (Cuadro 3).

Desarrollo ovárico. El desarrollo longitudinal de los ovarios de las hembras recuperadas de los tres diferentes frutos fue similar durante los primeros cinco días de edad. Después de cinco días de edad, la longitud del ovario fue mayor en las hembras recuperadas de mango "Ataulfo", registrando el mayor tamaño cuando las moscas alcanzaron 19 días de edad (Fig. 4 A), cuya diferencia con el desarrollo longitudinal de los ovarios registrado en las otras hembras fue significativa $(F=18.43$; g.l. $=2,12$; $P<0.001)$. Las hembras de los 19 hasta los 31 días de 


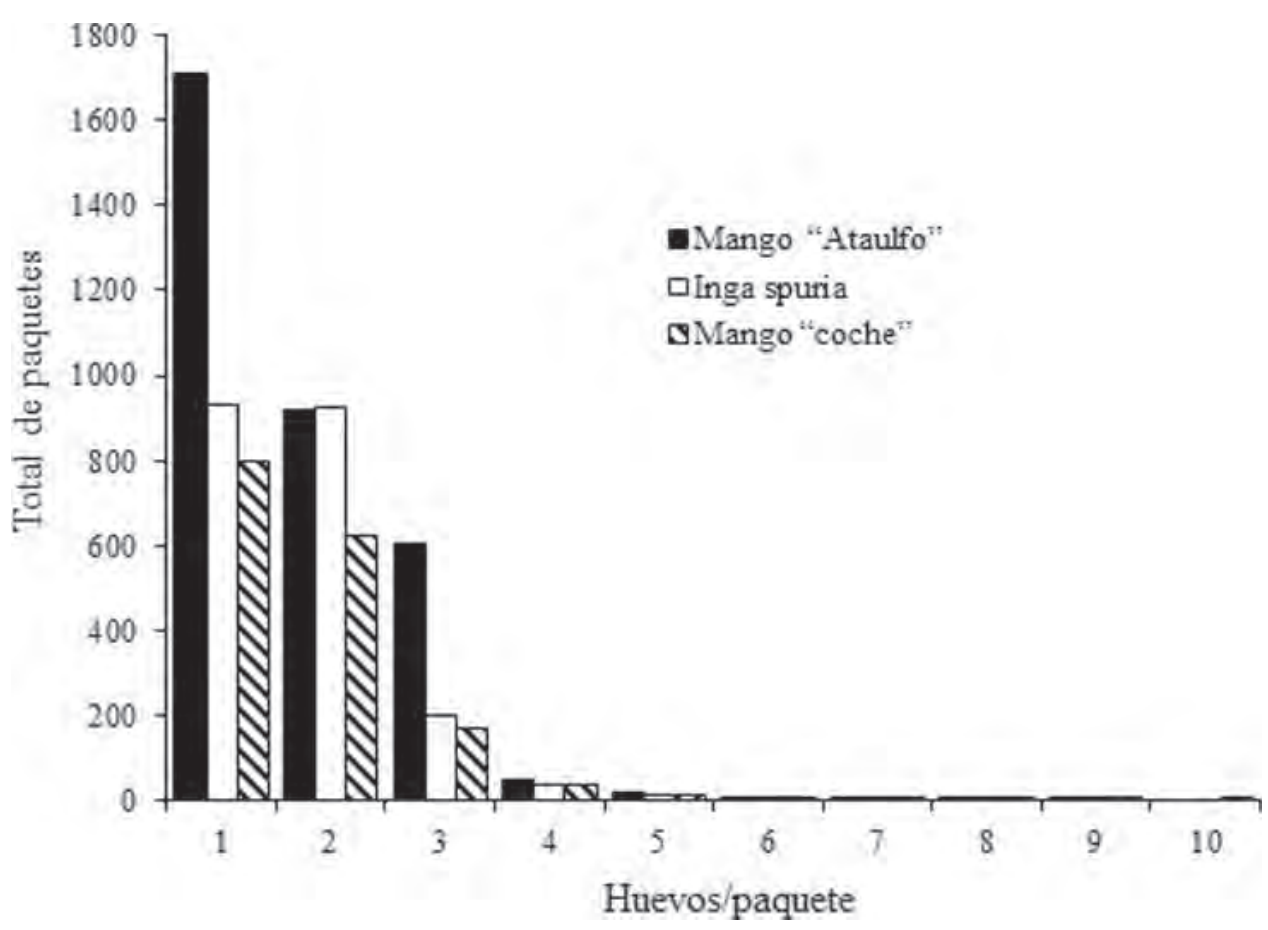

Figura 3. Cantidad de huevos por ovipostura por hembras de Anastrepha distincta criadas en su hospedero natural Inga spuria y en frutos de mango bajo condiciones de laboratorio.

Cuadro 3. Parámetros poblacionales de Anastrepha distincta provenientes de su hospedero natural Inga spuria y de frutos de mango infestados bajo condiciones de laboratorio.

\begin{tabular}{lccc}
\multicolumn{1}{c}{ Parámetro } & Mango “Ataulfo” & Mango “coche” & Inga spuria \\
\hline $\begin{array}{l}\text { Tasa intrínseca de crecimiento } \\
\text { (r) }\end{array}$ & 0.080 & 0.078 & 0.065 \\
Tasa finita de crecimiento $(\lambda)$ & 1.08 & 1.08 & 1.06 \\
Tiempo de duplicación (días) & 8.65 & 8.87 & 10.53 \\
Tiempo de Generación (TG) & 73.10 & 70.37 & 77.89 \\
\hline
\end{tabular}

edad, aunque con ligeras variaciones, registraron ovarios de longitudes similares.

En hembras de 10 días de edad el ancho del ovario fue significativamente de mayor tamaño en las que se recuperaron de mango "Ataulfo" $(F=22.0$; g.l. $=2$, $12 ; P$ $<0.001)$. Después de 10 días de edad se registraron ligeras variaciones con tendencias a una reducción hasta que alcazaron 25 días de edad (Fig. 4 B). Los ovarios de las hembras criadas en I. spuria presentaron un mayor número de ovariolas, mientras que en las hembras recuperadas de mango “coche” el número de ovariolas fue menor (Fig. 5).

\section{DISCUSIÓN}

La capacidad de $A$. distincta para infestar frutos de mango en condiciones de laboratorio fue confirmada por su desarrollo larvario en estos frutos, así como por los valores de fecundidad, fertilidad y parámetros de población encontrados. Las curvas de sobrevivencia obtenidas y los valores de expectativa de vida muestran que $A$. distincta es una especie longeva al compararse con A. ludens, $A$. obliqua y A. suspensa que son del mismo género (Liedo et al. 1992, Sivisnki 1993, Jaldo et al. 2007). Estos mismos autores indican que el hospedero larvario tiene un efecto en la mortalidad de los adultos, observándose una mayor supervivencia en los obtenidos del hospedero natural. Los valores de expectativa de vida de los adultos que se obtuvieron de los 3 tratamientos (rango: 69 - 132 días) fueron similares o mayores a los que se ha reportado para otras especies de Anastrepha en condiciones similares. Por lo que se han reportado rangos de 54 a 79 días para A. obliqua, 110 días para $A$. ludens y 75 días para $A$. serpentina (Liedo et al. 1992), y de 73 días para A. suspensa (Sivinski 1993).

En el caso de la fecundidad sucedió lo contario, las hembras procedentes de mango pusieron una mayor can- 

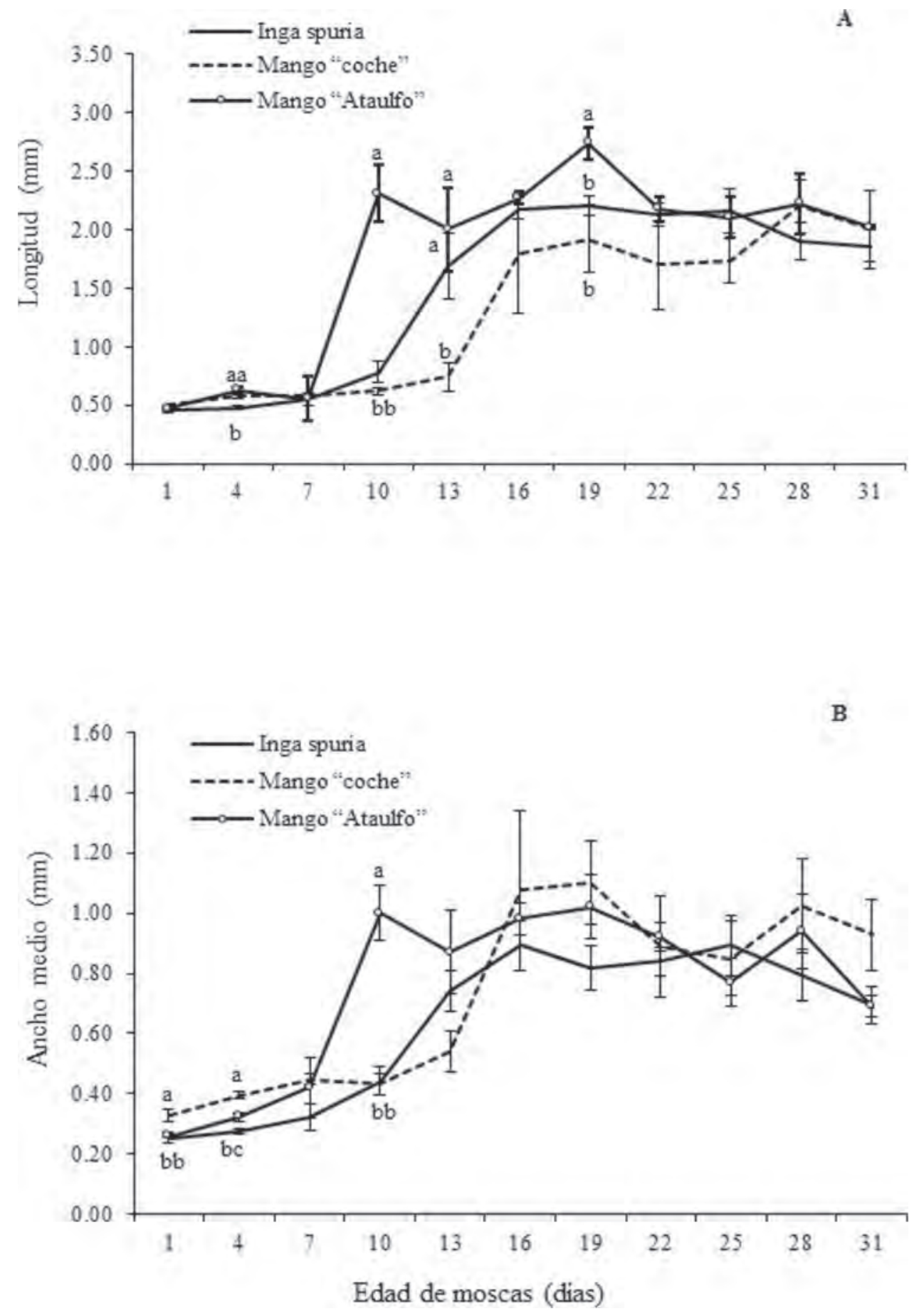

Figura 4. Longitud (A) y ancho medio (B) del ovario izquierdo de hembras de Anastrepha distincta criadas en su hospedero natural Inga spuria y en frutos de mango bajo condiciones de laboratorio ( $N=6$ hembras/tratamiento/edad).

tidad de huevos que las provenientes de I. spuria. Esta mayor fecundidad de las criadas en mango puede explicar su menor sobrevivencia, como "costo de la reproducción” (Carey et al. 2008). Las hembras de A. distincta registraron su mayor fecundidad entre los 25 y 40 días de edad, con una producción máxima a los 30 días de edad. En promedio pusieron 1.3, 3.6 y 5.8 huevos por hembra por día (h/h/d) para mango “coche”, mango "Ataulfo” e I. spuria, respectivamente. La fecundidad de A. distincta puede ser considerada como intermedia comparada con $10.8 \mathrm{~h} / \mathrm{h} / \mathrm{d}$ reportada para $A$. ludens, y $15.0 \mathrm{~h} / \mathrm{h} / \mathrm{d}$ para $A$. obliqua (Liedo et al. 1992), 2.5 y $0.6 \mathrm{~h} / \mathrm{h} / \mathrm{d}$ para hembras de $A$. fraterculus que se desarrollaron en guayaba (Psidium guajava L.) y manzana (Malus domestica L.), respectivamente 


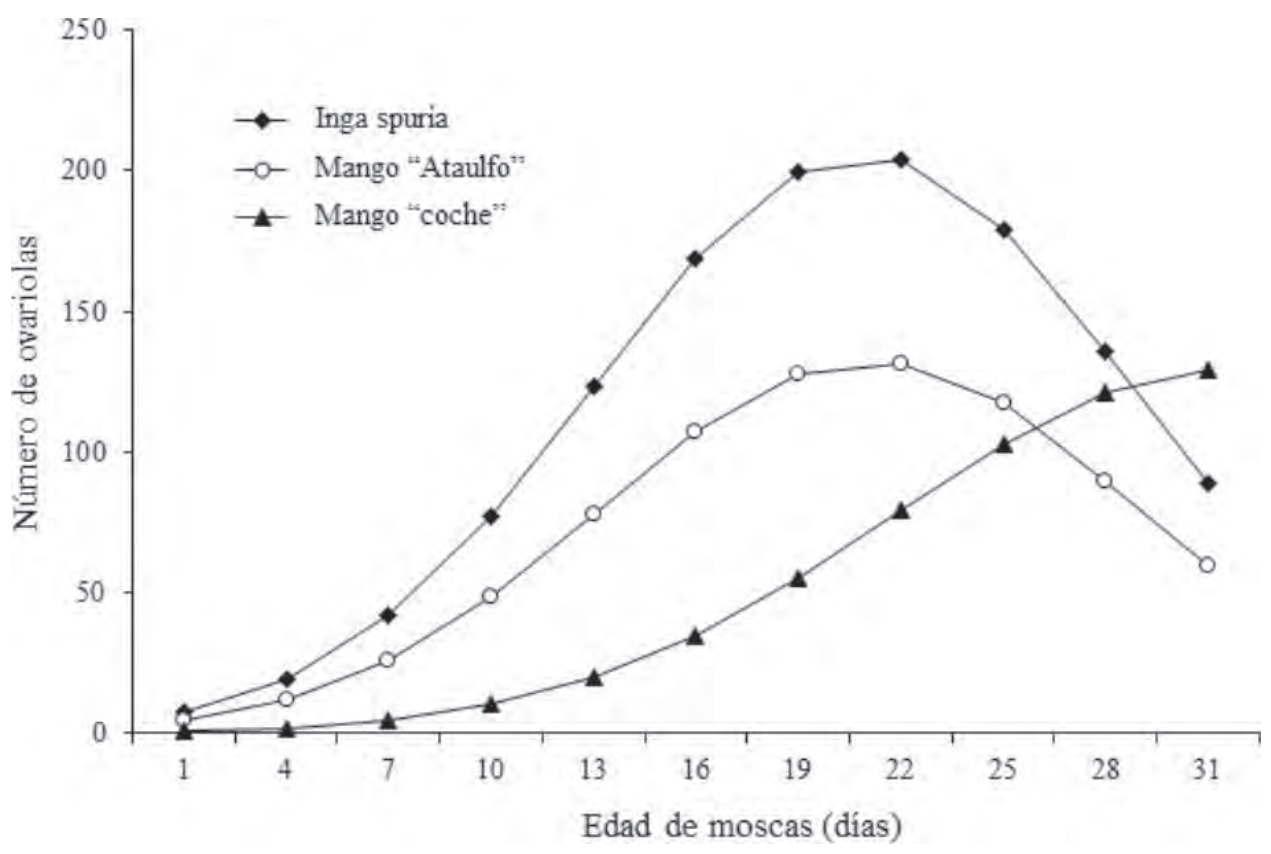

Figura 5. Cantidad de ovariolas en hembras de Anastrepha distincta criadas en su hospedero natural Inga spuria y en frutos de mango bajo condiciones de laboratorio $(N=6$ hembras/tratamiento/edad).

(Sugayama et al. 1998). Estos valores de fecundidad y la similitud de los valores de fertilidad entre los tratamientos, sugiere que el mango no tuvo un efecto adverso sobre la producción de huevos, por el contario, que las larvas pudieron alimentarse y desarrollarse satisfactoriamente, resultando en adultos aptos, capaces de producir huevos viables.

Los parámetros demográficos determinados en la población muestran que $A$. distincta tiene la posibilidad de desarrollar poblaciones de rápido crecimiento cuando utiliza frutos de mango para su desarrollo larvario. Las tasas de crecimiento, el tiempo de generación y el tiempo en que se duplica la población caen dentro de los rangos reportados para otras especies de Anastrepha de importancia económica (Liedo et al. 1993); este hecho indicó que los adultos de $A$. distincta no se vieron limitados en su aptitud.

El número de ovariolas que se observaron no fue un buen indicador para reflejar una mayor producción de huevecillos, ya que mientras el número de ovariolas fue menor en las hembras de mango "coche", estas fueron las que más huevos produjeron. Este hecho sugiere que no todos los oocitos que se producen en la zona germinal de los ovarios de las hembras llegaron a su máximo desarrollo (huevo). Lo anterior puede ser atribuido a las reservas adquiridas durante la alimentación larvaria, ya que la dieta como adultos fue la misma para los tres tratamientos.
Nuestros resultados indican que $A$. distincta tiene posibilidad para infestar mango en condiciones de laboratorio y producir poblaciones con potencial de crecimiento a partir de este hospedero. ¿Por qué entonces no sucede? ¿Por qué siendo una especie que se localiza en áreas donde hay árboles de mango en simpatría con árboles de Inga spp., no se le encuentra infestando estos frutos? Como posibles causas podemos señalar el no reconocimiento a distancia de los frutos de mango como hospederos (por estímulos visuales y/o químicos), la competencia con otras especies como A. ludens y A. obliqua que sí utilizan el mango como hospedero, o la presencia de latex u otros mecanismos de resistencia de los frutos de mango cuando están en el árbol.

La detección de frutos hospederos a distancia requiere de estímulos visuales y químicos a los que las especies están adaptadas (Prokopy \& Owen 1983). Bajo las condiciones de nuestro estudio, las hembras no requirieron hacer la búsqueda del recurso y no tuvieron otras opciones. Bajo condiciones naturales, si no existen las señales que indiquen a las hembras que el mango es un recurso para ovipositar, seguramente no lo utilizarán. La competencia interespecífica ha sido propuesta como mecanismo de partición de nichos o utilización de recursos (Atkinson \& Shorrocks 1981, Reitz \& Trumble 2002) y las moscas de la fruta no han sido la excepción (Aluja \& Diaz-Fleischer 2006, Duyck et al. 2004, Duyck et al. 2008). Pero existe poca evidencia empírica que confir- 
me a las predicciones teóricas (Kaplan \& Denno 2007). Bajo condiciones naturales un porcentaje variable de frutos de mango no son infestados cuando no se aplican medidas de control, por lo que consideramos que la explicación de la competencia interespecífica es poco probable.

La susceptibilidad de un fruto para ser infestado por moscas de la fruta está asociada al grado de madurez y al contenido de latex (Houston 1981, Seo et al. 1983, Greany et al. 1985). En este estudio, la infestación de frutos de ambos cultivares de mango fue en condiciones forzadas de laboratorio, lo cual, aunque no se determinó, se puede argumentar que el contenido de latex al momento de ser infestados había disminuido y por consecuencia la tolerancia de ser infestados. La susceptibilidad o tolerancia de las variedades de mango a la infestación, incluso por especies que comúnmente infestan este fruto, es variable (Aluja et al. 2014). Estas tres causas no son mutuamente excluyentes y requieren ser investigadas para poder determinar el potencial de A. distincta para ampliar su rango de hospederos. La evidencia del caso de $A$. fraterculus infestando manzanas en el sur de Brasil (Sugayama et al. 1998), y de A. obliqua infestando carambola (Averrhoa carambola L.), en Sao Paulo, Brasil (Souza-Filho et al. 2000), indican claramente que existe el potencial de estas especies para ampliar su rango de hospederos.

Anastrepha distincta es una especie que actualmente no tiene importancia como plaga agrícola, por lo que ha sido poca estudiada. La información reportada aquí descarta que no sea plaga del mango por incompatibilidad entre las dos especies o por resistencia de la especie vegetal, y demuestra el potencial que tiene para colonizar nuevos hospederos. El cambio climático, la eliminación de hospederos preferenciales, la reducción en las poblaciones de enemigos naturales y la eliminación de la competencia por la supresión de otras de especies de moscas de la fruta, son factores que eventualmente podría favorecer ese potencial para ampliar su rango de hospederos. Sin embargo, estos resultados no deben ser utilizados como argumento para establecer medidas regulatorias al mango por la presencia de $A$. distincta, pues nuestras infestaciones fueron bajo condiciones de laboratorio y los datos de campo demuestran que la probabilidad de infestación del mango por esta especie es prácticamente nula.

Agradecimientos. A V. Hernández-Ortiz y a dos revisores anónimos por sus valiosas sugerencias que contribuyeron a mejorar la calidad del trabajo. A Pablo Montoya (Programa Moscafrut SAGARPA-IICA) por sus valiosos comentarios a la primera versión. A Gustavo Rodas, Sandra L. Rodríguez y Ezequiel de León, por su apoyo técnico para la formación de las colonias e infestación de frutos.

\section{LITERATURA CITADA}

Aluja, M. 1994. Bionomics and management of Anastrepha. Annual Review of Entomology, 39: 155-178.

Aluja, M. \& Díaz-Fleischer, F. 2006. Foraging behavior of Anastrepha ludens, A. obliqua, and A. serpentina in response to feces extracts containing host marking pheromone. Journal of Chemical Ecology, 32: 367-389.

Aluja, M, Arredondo, J., Diaz-Fleischer, F., Birke, A., Rull, J., Niogret, J. \& Epsky, N. 2014. Susceptibility of 15 mango (Sapindales: Anacardaceae) cultivars to the attack by Anatrepha ludens and Anatrepha obliqua (Diptera: Tephritidae) and the role of underdeveloped fruit as pest reservoirs: Management implications. Journal of Economic Entomology, 107: 375-388.

Atkinson, W.D. \& Shorrocks, B. 1981. Competition on a divided and ephemeral resource: A simulation model. Journal of Animal Ecology, 50: 461-471.

Boller, E. F. 1968. An artificial oviposition device for the European cherry fruit fly, Rhagoletis cerasi. Journal of Economic Entomology, 61: 850-852.

Carey, J.R. 1984. Host-specific demographic studies of the Mediterranean fruit fly Ceratitis capitata. Ecological Entomology, 9: 261-270.

Carey, J. R. 1993. Applied demography for biologists. New York, Oxford. 206 pp.

Carey, J.R., Harshman, L.G., Liedo, P., Müller, H. G., Wang, J.L. \& Zhang, Z. 2008. Longevity-fertility trade-offs in the Tephritid fruit fly, Anastrepha ludens, across dietary-restriction gradients. Aging Cell, 7: 470-477.

Celedonio-Hurtado, H., Liedo, P., Aluja, M., Guillén, J., Berrigan, D. \& Carey, J. 1988. Demography of Anastrepha ludens, A. obliqua and A. serpentina (Diptera: Tephritidae) in Mexico. Florida Entomologist, 71: 111-119.

Duyck, P.F., David, P. \& Quilici, S. 2004. A review of relationships between interspecific competition and invasions in fruit flies (Diptera: Tephritidae). Ecological Entomology, 29: 511-520.

Duyck, P.F., David, P., Pavoine, S. \& Quilici, S. 2008. Can host-range allow niche differentiation of invasive polyphagous fruit flies (Diptera: Tephritidae) in La Réunion? Ecological Entomology, 33: 439-452.

Greany, P. D., Shaw, P. E., Davis, P. L. \& Hatton, T. T. 1985. Senescence-related susceptibility of marsh grapefruit to laboratory infestation by Anastrepha suspensa (Diptera: Tephritidae). Florida Entomologist, 68: 144-150.

Hernández-Ortiz, V. 1992. El género Anastrepha Schiner en México (Diptera: Tephritidae): Taxonomía, distribución y sus plantas huéspedes. Instituto de Ecología, A. C. y Sociedad Mexicana de Entomología. Xalapa, Ver. México. 162 pp.

Houston, W. W. K. 1981. Fluctuation in numbers and the significance of the sex ratio of the Mexican fruit fly, Anastrepha ludens caught in Mc Phail traps. Entomologia Experimentalis et Applicata, 30: 140-150.

Jaldo, H. E., Willink, E. \& Liedo, P. 2007. Demographic analysis of mass-reared Anastrepha fraterculus (Diptera: Tephritidae) in Tucumán, Argentina. Revista Industrial y Agrícola de Tucumán, 84: $15-20$.

Kaplan, I. \& Denno, R.F. 2007. Interspecific interactions in phytophagous insects revisited: A quantitative assessment of competition theory. Ecology Letters, 10: 977-994.

Krainacker, D.A., Carey, J.R. \& Vargas, R.I. 1987. Effect of larval host on life history traits of the Mediterranean fruit fly, Ceratitis capitata. Oecologia, 73: 583-590. 
Liedo P., Carey, J.R., Celedonio, H. \& Guillén, J. 1992. Size specific demography of three species of Anastrepha fruti flies. Entomologia Experimentalis et Applicata, 63: 135-142.

Liedo, P. \& Carey, J. R. 1996. Demography of fruit flies and implications to action programs, pp. 299-308. In: B. A. McPheron \& G. J. Steck (Eds). Fruit fly pests: A world assessment of their biology and management. St. Lucie Press, Delray Beach, Fl., USA.

Liedo, P., Carey, J.R., Celedonio, H. \& Guillén, J. 1993. Demography of Anastrepha fruit flies: A case study of three species of economic importance, pp. 115-124. In: M. Aluja and P. Liedo (Eds). Fruit flies. Biology and management. Springer-Veralg, New York.

Ni,. W. L., Li, Z. H., Chen, H. J., Wan, F. H., Qu, W. W., Zhang, Z. \& Kriticos, D. J. 2012. Including climate change in pest risk assessment: The peach fruit fly, Bactrocera zonata (Diptera: Tephritidae). Bulletin of Entomological Research, 102: 173-183.

Norrbom, A.L. \& Chung-Kim, K. 1988. A list of the reported host plants of the species of Anatrepha (Díptera: Tephritidae). USDA, APHIS, PPQ. APHIS, 81-52.

Norrbom, A. L., Zucchi, R. A. \& Hernández-Ortiz, V. 1999. Phylogeny of the genus Anastrepha and Toxotrypana (Trypetinae: Toxotrypanini) based on morphology, pp. 299-342. In: M. Aluja and A. L. Norrbom. (Eds). Fruit flies (Tephritidae): Phylogeny and evolution of behavior. CRC Press, Boca Raton. Florida, USA. $944 \mathrm{pp}$.
Prokopy, R.J. \& Owens, E.D. 1983. Visual detection of plants by herbivorous insects. Annual Review of Entomology, 28: 337-364.

Reitz, S. \& Trumble, J.T. 2002. Competitive displacement among insects and arachnids. Annual Review of Entomology, 47: 435-465.

R Core Team. 2014. R: A language and environment for statistical computing. R Foundation for Statistical Computing, Vienna, Austria. URL http://www.R-project.org/.

SAS Institute. 1999. SAS/STAT user's guide. Versión 8 ed. SAS Institute, Cary, NC.

Seo, S.T., Tang, C.S., Sanidad, S. \& Takemaka, T.H. 1983.Hawaiian fruit flies (Diptera: Tephritidae): Variation of index of infestation with benzyl isothiocyanate concentration and color of maturing papaya. Journal of Economic Entomology, 76: 535-538.

Sivinski, J. M. 1993. Longevity and fecundity in the Caribbean fruit fly (Diptera: Tephritidae): Effects of mating, strain and body size. Florida Entomologist, 76: 635- 644.

Souza-Filho, M. F., Raga, A. \& Succhi, R. A. 2000. Incidencia de Anastrepha obliqua (Macquart) y Ceratitis capitata (Wiedemann) (Diptera: Tephritidae) en carambola (Averrhoa carambola L.) en ocho localidades del Estado de Sâo Paulo, Brasil. Anais da Sociedade Entomologica do Brasil, 29: 367-371.

Sugayama, R. L., Kovaleski, A., Liedo, P. \& Malavasi, A. 1998. Colonization of a new fruit crop by Anastrepha fraterculus (Diptera: Tephritidae) in Brazil: A demographic analysis. Environmental Entomology, 27: 642-648. 